\title{
Psychische Krisen bei Kindern und Jugendlichen
}

\author{
Mental Crises in children and adolescents
}

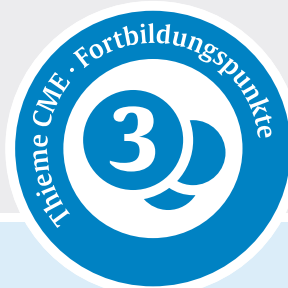

Michael Frey

Technische Hochschule Deggendorf und Klinik und

Poliklinik für Kinder- und Jugendpsychiatrie, Psychosomatik und Psychotherapie der LMU München

\section{ZUSAMMENFASSUNG}

Psychische Krisen im Kindes- und Jugendalter sind geprägt von altersspezifischen Vulnerabilitäten. Im Kindesalter stehen sie oft im Zusammenhang mit dem ausgeprägten Abhängigkeitsverhältnis gegenüber den Eltern und Situationen, in denen
Eltern durch eine eigene oder eine psychische Erkrankung ihres Kindes in eine Überforderungssituation geraten. Im Jugendalter interagieren neurobiologische Veränderungen und herausfordernde Entwicklungsaufgaben, die eine krisenhafte Zuspitzung begünstigen können. Im Hinblick auf Kriseninterventionsstrategien für diese Altersgruppen stellen sich vor allem die Fragen, wie der Zugang zur Krisenintervention gelingt und welche Strategien es für die wichtigen sozialen Bezugssysteme Familie und Schule gibt?

\section{Krisensituationen in Abhängigkeit vom Entwicklungsalter}

Ausgehend von einer Krisendefinition, die sich auf Ereignisse und Lebensumstände bezieht, welche die individuell verfügbaren Bewältigungsmöglichkeiten übersteigen und damit zu einem als bedrohlich erlebten seelischen Ungleichgewicht führen, sind sowohl Umgebungseinflüsse als auch in der Person liegende Faktoren zu berücksichtigen [1]. Im Sinne einer Wechselseitigkeit konstelliert sich bei Kindern und Jugendlichen dabei vieles in der Eltern-Kind-Beziehung. Die Interaktion und Kommunikation zwischen Eltern und ihren Kindern spielt daher im Krisenverständnis und in der Krisenintervention bei Minderjährigen eine wichtige Rolle und markiert einen der wesentlichen Unterschiede zum Erwachsenenalter.

In Bezug auf die psychosoziale Entwicklung ist ein wesentlicher Entwicklungsstrang für das Verständnis von psychischen Krisen im Kindes- und Jugendalter der von der Abhängigkeitsbeziehung hin zur Autonomie. Je jünger die Kinder, desto größer ist das Abhängigkeitsverhältnis von den primären Bezugspersonen, im Regelfall den Eltern. Mit zunehmendem Alter wird mehr Selbstständigkeit und Unabhängigkeit gewonnen und mit dem Einsetzen der Pubertät wird die Autonomieentwicklung zu einem der Hauptmotive in der Adoleszenz. Krisen im Kindesalter stehen daher häufig im Zusammenhang mit der großen Abhängigkeit und der daraus resultierenden Schutzbedürftigkeit, der auch die Gesellschaft durch rechtliche Vorschriften und Interventionsmöglichkeiten zum Schutze des Kindeswohls Rechnung trägt. Im Jugendalter hingegen dominieren meist Krisenkonstellationen, die einen Zusammenhang mit den alterstypischen Entwicklungsaufgaben vor dem Hintergrund der Autonomieentwicklung aufweisen. Der Ablösungsprozess von den Eltern hin zur sozialen Integration in die Gruppe der Gleichaltrigen und die damit verbundenen Konflikte sind häufige Anlässe für krisenhafte Zuspitzungen. Die einzelnen Lebens- und Entwicklungsphasen bringen dabei spezielle Vulnerabilitäten für psychische Erkrankungen mit sich.

\section{Prävalenz}

Hinsichtlich der Prävalenz von psychischen Krisen im Kindes- und Jugendalter können nur orientierende Angaben herangezogen werden, da es zum einen an einer einheitlichen Definition mangelt und zum anderen das Setting, in dem die Krisen auftreten, sehr unterschiedlich sein kann. So zeigen Studien, dass in den USA ca. $7 \%$ der in pädiatrischen Notaufnahmen behandelten Kinder und Jugendlichen, meist zwischen 12 und 17 Jahren, einen psychiatrischen Vorstellungsgrund hatten und die Anzahl der Kinder und Jugendlichen, die sich dort mit psychischen Problemen, vor allem Suizidalität und Aggressivität, vorstellten, sich zwischen 2000 und 2016 fast verdoppelt hatte [2, 3]. Für Deutschland belegen Daten aus dem Jahr 2013, dass ca. $40 \%$ der Aufnahmen in kinder- und jugendpsychiatrischen Kliniken ungeplant im Rahmen einer Notfallvorstellung erfolgten. Auch hier konnte über die Jahre ein deutlicher Anstieg beobachtet werden [4]. Ein weiterer Marker für Krisen- und Gefährdungssituationen sind Inobhutnahmen durch Jugendämter. Lässt man die Inobhutnahmen der unbegleitet einreisenden Minderjährigen, die in den letzten Jahren sukzessiv abnimmt, außer Acht, stieg die Anzahl der Inobhutnahmen aus anderen Gründen weiter an, zwischen 2009 und 2019 um ca. 30\% [5]. Für die aktuelle Situation während der Pandemie zeigen die noch spärlich vorliegenden Erhebungen, dass die psychische Belastung bei Minderjährigen im Vergleich zu Zeitpunk- 
ten vor der Pandemie gestiegen ist (z. B. COPSY-Studie) [6]. Kinder- und jugendpsychiatrische Praxen und Kliniken berichten von einer Zunahme von krisenhaften Zuspitzungen. Insbesondere internalisierende Störungen wie Ängste, Depressionen und damit einhergehende Suizidalität sowie Essstörungen scheinen deutlich zugenommen zu haben [7]. Zugleich ist der Zugang zum Hilfesystem aus Kapazitätsgründen und pandemiebedingten Einschränkungen, die sowohl installierte Jugendhilfemaßnahmen beeinträchtigen als auch zu einem Wegfall von Hinweisgebern (z. B. Erzieher, Lehrer) im Kinderschutz führen, erschwert [7].

\section{Psychische Krisen im Kindesalter}

Für die Art von Gefährdungs- und Krisensituationen im Kindesalter können vor allem statistische Angaben zu Gründen von Inobhutnahmen einen Hinweis geben. Bei Inobhutnahmen von Kindern $\leq 9$ Jahren im Jahr 2019 wurden in über der Hälfte der Fälle eine Überforderung der Eltern und in mehr als einem Drittel der Fälle Anzeichen für Vernachlässigung als Gründe genannt [5]. Die entwicklungsbedingte hohe Abhängigkeit der Kinder stellt hier einen Risikofaktor dar. Eine besonders vulnerable Gruppe sind dabei Familien mit mindestens einem psychisch kranken Elternteil. Vor dem Hintergrund der eigenen psychischen Belastung können die Bedarfe der Kinder durch die Eltern oftmals nicht ausreichend adressiert werden. Schätzungen zufolge wachsen in Deutschland ca. 2-3 Mio. Kinder mit zumindest einem psychisch erkrankten Elternteil auf und ca. 10-30\% der stationär psychiatrisch behandelten Erwachsenen haben minderjährige Kinder $[8,9]$. Kinder psychisch kranker Eltern haben selbst ein deutlich erhöhtes Risiko, später eine psychische Erkrankung zu entwickeln [9]. Neben genetischen Faktoren spielen auch Belastungen, welche die psychische Vulnerabilität der Eltern mit sich bringt, eine Rolle. Dabei konnte eine retrospektive qualitative Studie 4 Hauptbelastungsfaktoren herausarbeiten: Orientierungslosigkeit, da es den Kindern nicht immer gelingt die Verhaltensweisen der Eltern einzuordnen, Schuldgefühle, da sich die Kinder für das Befinden der Eltern verantwortlichen fühlen sowie die erlebte Verpflichtung zum Schweigen und Isolation, da Kinder den Eindruck haben, das Erlebte und ihre Sorgen diesbezüglich nicht außerhalb der Familie besprechen zu können [10]. Die Versorgungsforschung zeigt, dass der Zugang zum Versorgungssystem insbesondere für Kinder mit einem hohen Risiko, z. B. aufgrund von Vernachlässigung, unzureichend ist. Sie erhalten entweder keine Behandlung oder diese erfolgt mit großer Verzögerung, da die Betroffenen nicht oder nicht rechtzeitig identifiziert und an die zuständige Stelle weitergeleitet werden [4]. Vor diesem Hintergrund kommt in dieser Altersgruppe den Behandlern und professionellen Helfern im Rahmen von Kriseninterventionen bei den Eltern eine besondere Verantwortung zu, die Kinder und deren Bedarfe im Blick zu haben und den Eltern Unterstützung anzubieten, um sie in ihrer Elternrolle zu stärken.
Eine weitere häufige Konstellation, die auch in einem sonst von psychiatrischen Erkrankungen unbelasteten Elternhaus zu Krisen führen kann, besteht, wenn Kinder an externalisierenden Störungen wie ADHS (Aufmerksamkeitsdefizit/Hyperaktivitätsstörung) oder einer Störung des Sozialverhaltens leiden und die Eltern in ihren pädagogischen Möglichkeiten überfordert sind [11]. Ebenso können die Besonderheiten von Kindern mit Autismusspektrumsstörungen Familien an die Grenzen ihrer Belastbarkeit bringen [12]. Auch in diesen Fällen geht es neben einer adäquaten Behandlung für die betroffenen Kinder in der Regel darum, Unterstützungsmöglichkeiten für die Familien zu finden, um die Eltern-Kind-Interaktion günstig zu beeinflussen.

\section{Krisen und psychische Erkrankungen im Jugendalter}

Die Adoleszenz birgt besondere Risiken für krisenhafte Zuspitzungen. Dabei interagieren neurobiologische Reifungsprozesse und für das Alter typische Entwicklungsaufgaben, die das Auftreten bestimmter psychischer Erkrankungen begünstigen. So ist die Adoleszenz die Lebensphase mit einer stark kumulierten Inzidenz von psychischen Erkrankungen [13]. Inzidenzen für Abhängigkeitserkrankungen und nicht suizidales selbstverletztendes Verhalten (NSSV) zeigen einen starken Anstieg ab der Pubertät, ebenso depressive Störungen, vor allem bei Mädchen $[14,15]$. Auch psychotische Störungen manifestieren sich in der Regel erstmals im spätadoleszenten Alter $[16,17]$. Neuropsychiatrische Erkrankungen sind weltweit der häufigste Grund für mit chronischen Erkrankungen und Behinderung verbrachte Jahre in der Altersgruppe zwischen 10 und 24 Jahren [13].

\section{Neurobiologische Reifungsprozesse der Adoleszenz}

Mit Einsetzen der Pubertät kommt es zu zerebralen Reifungsprozessen, die sich bis ins 25. Lebensjahr ziehen. Während die weiße Substanz durch sukzessive Myelinisierung von der Kindheit bis zum Erwachsenenalter kontinuierliche an Volumen zunimmt, ist dies bei der grauen Substanz anders; durch „Pruning“ kommt es in der Adoleszenz zu einer massiven Abnahme der Synapsendichte [16]. Zudem entsteht durch hormonelle Veränderungen ausgelöst ein gewisses Ungleichgewicht zwischen früher reifenden subkortikalen Strukturen (v. a. des limbischen Systems) und erst deutlich später reifenden präfrontalen Arealen, die z. B. zur Emotionsregulation, Risikoabschätzung, Lenkung von Impulsen im Sinne mittel- und langfristiger Ziele oder sozialer Erfordernisse dienen. Diese Konstellation ist die neurobiologische Grundlage der im Jugendalter zu beobachtenden erhöhten Empfänglichkeit für Belohnungsreize jeder Art, den Schwierigkeiten in der Emotionsregulation sowie einer vermehrten Impulsivität und ihr werden entwicklungsfördernde Eigenschaften (z. B. ausgeprägte Flexibilität, Förderung der Autonomie- 
entwicklung, Offenheit für Neues) nachgesagt. Sie bringt jedoch auch eine besondere Vulnerabilität für psychiatrische Erkrankungen und dysfunktionale Verhaltensweisen mit sich, z. B. für Abhängigkeitserkrankungen aufgrund der großen Empfänglichkeit für Belohnungsreize oder für NSSV, als dysfunktionale Strategie zur Emotionsregulation $[16,18,19]$.

\section{Entwicklungsaufgaben der Adoleszenz}

Beginnend mit der Pubertät sehen sich Jugendliche und ihr Umfeld konfrontiert mit teils massiven Veränderungen und Entwicklungsimpulsen. Neben den körperlichen Veränderungen, die den Boden bereiten für erste psychosexuelle Erfahrungen, sind die Themen Identität und Autonomie gewissermaßen Leitmotive der Entwicklung. Die Identitätsentwicklung interagiert mit der Ablösung vom Elternhaus und der vermehrten Hinwendung zu einer Gruppe Gleichaltriger. Die damit einhergehenden Abgrenzungsversuche von den Eltern einerseits und das Bedürfnis nach Zugehörigkeit und Akzeptanz zu einer Gruppe Gleichaltriger andererseits sind dabei Herausforderungen, an denen auch immer ein Scheitern möglich ist und was zu einer krisenhaften Zuspitzung führen kann. Erlebnisse und Lebensereignisse, die aus Erwachsenenperspektive ggf. nicht die verstörende Tragweite haben, können für Jugendliche existenziell bedrohlich wirken, da vieles „das erste Mal“ ist und ein biografischer Erfahrungshorizont, vor dem das Erlebte relativiert werden könnte, fehlt. Ein besonders sensibler Entwicklungsbereich ist dabei die soziale Integration. Das Fehlen eines Zugehörigkeitsgefühls ist vor allem im Jugendalter ein wesentlicher Risikofaktor für psychische Krisen und psychiatrische Erkrankungen. Netzwerkanalysen zeigen beispielsweise, dass im Gegensatz zu Erwachsenen bei Jugendlichen empfundene „Einsamkeit“ ein zentrales Symptom in der Psychopathologie depressiver Erkrankungen ist und Ausgrenzungserfahrungen wie Mobbing und Cyberbullying erhöhen das Suizidrisiko bei Minderjährigen beträchtlich; eine Metaanalyse zeigte eine Verdoppelung des Risikos für Suizidgedanken und Suizidversuche [20, 21].

\section{Suizidalität im Jugendalter}

Suizidalität als Ausdruck einer existenziellen Zuspitzung einer psychischen Krise spielt insbesondere im Jugendalter eine wesentliche Rolle. Wenngleich die Suizidprävalenz bei Minderjährigen mit ca. 200 Suiziden/Jahr in Deutschland niedrig ist, stellt Suizid in den meisten westlichen Ländern die zweit- bis dritthäufigste Todesursache bei Jugendlichen dar [22-24]. In deutschen Schulstudien geben fast ein Fünftel der jugendlichen Mädchen an, jemals Suizidgedanken gehabt zu haben und ein Zehntel einen Suizidversuch in der Vergangenheit unternommen zu haben; Jungen jeweils halb so häufig [25]. Eine großangelegte europäische Studie, die Jugendliche (15-16 Jahre) zu Suizidversuchen in der Vorgeschichte befragte, berichtet einen Median der Lebenszeitprävalenz von 10,5\% [26]. 15-30\% unternahmen innerhalb eines Jahres einen erneuten Suizidversuch. Das höchste Risiko dafür bestand in den ersten 1-4 Wochen nach der Entlassung aus einem stationären psychiatrischen Setting [27]. Als 3 der wichtigsten Risikofaktoren für Suizidversuche und vollendete Suizide im Jugendalter sind zu nennen: eine psychiatrische Erkrankung (v. a. Depression, Angststörungen und Substanzabusus), Suizidversuche in der Vorgeschichte und NSSV [23]. Aber auch ein fehlendes soziales Zugehörigkeitsgefühl und familiäre Konflikte stellen einen der nicht zu unterschätzenden Risikofaktoren für Suizidalität im Jugendalter dar [27]. Insbesondere in der Situation der Corona-Pandemie ist zu beobachten, dass die erzwungene Nähe der Familien (Homeschooling, Ausgangssperren etc.) auch dazu beiträgt, dass familiäre Konflikte vermehrt eskalieren, bis hin zu körperlichen Übergriffen, worauf auch erste internationale Studien hinweisen [28, 29]. Zeitgleich sind die Kontaktmöglichkeiten zu Gleichaltrigen eingeschränkt. Die COPSY-Studie zeigte für Deutschland, dass sich die Lebensqualität der Kinder und Jugendlichen verschlechtert und die Anzahl von psychischen Auffälligkeiten im Vergleich mit den Ergebnissen der BELLA-Studie vor der Pandemie ca. verdoppelt hat. Auch hier zeigte sich, dass Konflikte in der Familie zunahmen und häufiger eskalierten [6].

\section{Besonderheiten in der Krisenintervention}

Mit Blick auf die Krisenintervention stellen sich vor allem 2 Fragen: Wie ist der Zugang zu professioneller Hilfe und welche Vorgehensweisen in der Krisenintervention haben sich bewährt? Mit Blick auf evidenzbasierte Ansätze zur Krisenintervention bedarf es einer Differenzierung hinsichtlich der Indikation und des Settings. So besteht für spezielle Indikationen wie z. B. PTBS, Suizidalität oder NSSV einige Evidenz für spezielle therapeutische Ansätze [30, 31]. Hinsichtlich des Settings gibt es dabei vor allem Studien zu Kriseninterventionen in einem zumindest einige Termine umfassenden therapeutischen Rahmen sowie schulbasierte Interventionen. Wenig Evidenz gibt es für Settings, die mit sehr heterogenen Krisenformen konfrontiert sind und eine sehr kurze Interventionsdauer vorsehen, z. B. in pädiatrischen Notaufnahmen oder Interventionen vor Ort [3]. Im Folgenden soll nach der Frage, wie die Zugangswege zu professioneller Hilfe aussehen, kurz auf 2 der kinder- und jugendspezifischen Aspekte von Krisenintervention eigegangen werden: schulbasierte Ansätze und die Einbeziehung der Eltern bzw. Familie.

\section{Zugang zu professioneller Hilfe}

Die Möglichkeiten des Zuganges zu professioneller Hilfe sind altersabhängig. Im Kindesalter ist es die Verantwortung der Eltern, die Probleme ihrer Kinder zu erkennen, einzuordnen und ggf. die notwendige professionelle Hilfe hinzuzuziehen. In Fällen, in denen Eltern nicht dazu in der Lage oder willens sind, kommt dem Kinderschutz eine zentrale Bedeutung bei und damit auch Vertrauenspersonen und potenziellen Hinweisgebern in Gefährdungssituati- 
onen. Dies betrifft vor allem Erzieher und Lehrer. Die Situation der Corona-Pandemie, in der die Kinder auf ihr häusliches Umfeld zurückgeworfen sind, stellt daher gerade für die besonders gefährdeten Kinder ein hohes Risiko dar, dass notwendige Hilfen nicht in Anspruch genommen werden. Das wahre Ausmaß dieser Problematik wird wahrscheinlich erst nach den pandemisch bedingten Einschränkungen sichtbar werden.

Im Jugendalter verändern sich die Zugangswege und dem Hilfesuchverhalten der Jugendlichen selbst kommt eine zunehmend große Bedeutung bei. Dabei zeigen Studien, dass insbesondere Jugendliche mit internalisierenden Störungen nur zu einem geringen Teil (18-34\%) eine adäquate Behandlung erhielten [32]. Eine britische Studie zu NSSV ergab, dass ca. die Hälfte der betroffenen Jugendlichen Unterstützung zunächst bei Freunden suchte und an zweiter Stelle, mit jedoch nur 10-20\%, bei den Eltern [33]. Professionelle Unterstützung wurde von Jugendlichen selbst in unter $10 \%$ der Fälle gesucht [33]. Hürden Hilfe in Anspruch zu nehmen, bestehen vor allem in der Angst vor Stigmatisierung und Scham, unzureichendem Wissen über psychische Erkrankungen und dem Wunsch nach Autonomie und Eigenständigkeit [32].

\section{Schulbasierte Ansätze}

Schule als zentraler Bestandteil der Lebenswelt von Kindern und Jugendlichen ist mit Blick auf psychische Erkrankungen und Krisen in diesem Alter auch ein Ort für Aufklärung, Prävention und Krisenintervention. Ein Teil der Angebote adressiert dabei die genannten Hürden zur Inanspruchnahme professioneller Hilfen z. B. durch Informationsvermittlung und Aufklärung zu psychischen Erkrankungen und Suizidalität. Beides erwies sich in Studien als zielführend [34, 35]. Sekundärpräventive Ansätze, wie die Schulung sogenannter „Gatekeeper“ oder „Screening“-Maßnahmen z. B. zu Suizidalität, um Betroffene zu identifizieren und mit professioneller Hilfe in Kontakt zu bringen, zeigen bei heterogener Studienlage eher unzureichende Effekte [23, 34, 36]. Zudem stellen sich vor allem vor dem Hintergrund der oft niedrigen Spezifität der Screening-Instrumente auch ethische Fragen. Für ressourcenorientiert angelegte Vorgehensweisen, die z. B. das Verbundenheitsgefühl mit der Schule und soziale Kompetenzen fördern, konnte eine Reduktion von Suizidgedanken und Suizidversuchen bei Schülern belegt werden [37]. Eine wichtige Rolle, vor allem auch wegen des ausgeprägten „Werther-Effektes“ bei Jugendlichen, kommt auch der Postvention bei erfolgten Suiziden an Schulen zu. Dabei fehlt es an evidenzbasierten Vorgehensweisen, aber es existieren auf Expertenkonsens beruhende Empfehlungen [38].

\section{Familienbasierte Ansätze}

Wie zuvor anhand einiger Aspekte dargestellt, ist die Bedeutung des familiären Umfeldes und der Eltern-Kind-Beziehung für Krisen im Kindes- und Jugendalter essentiell.
Damit sind die familiären Bezüge auch ein wesentlicher Bestandteil von Kriseninterventionen in dieser Altersgruppe. Leider ist die Studienlage dazu sehr überschaubar und die meisten Untersuchungen liegen zum Thema Suizidalität vor. Es kann unterschieden werden zwischen Ansätzen, die sich primär auf die Eltern bzw. Eltern-Kind-Interaktion beziehen und Interventionen, welche die gesamte Familie in den Blick nehmen. Beide Vorgehensweisen erwiesen sich in Studien als erfolgreich. So konnten zur Suizidprävention bei Jugendlichen positive Effekte für Interventionen belegt werden, welche die Eltern-Kind-Interaktion als wesentliches Element beinhalteten oder als familienbasierte Kriseninterventionen angelegt waren [27, 31, 39]. Die Interventionen basieren dabei auf unterschiedlichen theoretischen Hintergründen von systemischen über bindungstheoretischen bis hin zu kognitiv-verhaltens-therapeutischen Konzepten [40]. Die Studienlage zu Wirkfaktoren ist unzureichend. Häufig adressieren die Interventionen jedoch Kommunikation und Interaktion der Familienmitglieder, beinhalten psychoedukative Elemente und beschäftigen sich mit Konflikt- und Problemlösungsstrategien [40]. Auch die S2k-Leitlinie zu Suizidalität im Kindes- und Jugendalter (2016) empfiehlt, dass psychotherapeutische Interventionen die Familie mit einbeziehen sollen [23].

\section{FAZIT FÜR DIE PRAXIS}

Im Kindesalter stehen Überforderungssituationen der Eltern als Grund für akute Krisen im Vordergrund. Eine besonders gefährdete Gruppe sind Kinder psychisch kranker Eltern. Damit kommt den professionellen Helfern der erkrankten Eltern eine zentrale Rolle im Kinderschutz zu. Entwicklungsbedingt sind Adoleszente besonders anfällig für die Erstmanifestation psychischer Erkrankungen und krisenhafte Zuspitzungen bis hin zu akuter Suizidalität. Familiäre Konflikte spielen dabei oft eine zentrale Rolle. Die Angst vor Stigmatisierung und unzureichendes Wissen über psychische Erkrankungen können eine Hürde für die Inanspruchnahme professioneller Hilfe sein. Als hilfreich haben sich - vor allem zur Suizidprävention - daher u. a. Ansätze erwiesen, welche die familiäre Interaktion und Kommunikation bzw. die Hürden zur Inanspruchnahme professioneller Hilfe adressieren. 


\section{Interessenkonflikt}

Erklärung zu finanziellen Interessen

Forschungsförderung erhalten: nein; Honorar/geldwerten Vorteil für Referententätigkeit erhalten: nein; Bezahlter Berater/interner Schulungsreferent/Gehaltsempfänger: nein; Patent/Geschäftsanteile/Aktien (Autor/Partner, Ehepartner, Kinder) an Firma (Nicht-Sponsor der Veranstaltung): nein; Patent/Geschäftsanteile/Aktien (Autor/Partner, Ehepartner, Kinder) an Firma (Sponsor der Veranstaltung): nein.

Erklärung zu nicht finanziellen Interessen

Der Autor gibt an, dass kein Interessenkonflikt besteht.

\section{Korrespondenzadresse}

\section{Prof. Dr. med. Michael Frey}

Technische Hochschule Deggendorf

Dieter-Görlitz-Platz 1, 94469 Deggendorf, Deutschland

Tel. 0991/3615120

michael.frey@th-deg.de

\section{Literatur}

[1] Cullberg J. Krisen und Krisentherapie. Psychiat Prax 1978; 5: 25-34

[2] Hoffmann JA, Stack AM, Samnaliev M et al. Trends in visits and costs for mental health emergencies in a pediatric emergency department, 2010-2016. Academic pediatrics 2019; 19: 386-393

[3] Carubia B, Becker A, Levine BH. Child psychiatric emergencies: updates on trends, clinical care, and practice challenges. Current psychiatry reports 2016; 18: 41

[4] Fegert JM, Kölch M, Krüger U. Versorgung psychisch kranker Kinder und Jugendlicher in Deutschland-Bestandsaufnahme und Bedarfsanalyse. Tagung der APK vom 2018

[5] Bundesamt für Statistik. Statistiken der Kinder- und Jugendhilfe 2019. Im Internet: https://www.destatis.de/DE/Themen/Gesellschaft-Umwelt/Soziales/Kinderhilfe-Jugendhilfe/ Publikationen/Downloads-Kinder-und-Jugendhilfe/vorlaeufige-schutzmassnahmen-5225203197004.pdf?_blob=publicationFile; Stand: 29.05.2021

[6] Ravens-Sieberer U, Kaman A, Otto C et al. Seelische Gesundheit und psychische Belastungen von Kindern und Jugendlichen in der ersten Welle der COVID-19-PandemieErgebnisse der COPSY-Studie. Bundesgesundheitsblatt-Gesundheitsforschung-Gesundheitsschutz 2021; 1-10

[7] Clemens V, Fegert J. Ein Marschallplan für Kinder, Jugend und Familie. Corona und die Folgen nicht einfach nur hinnehmen. Nervenheilkunde 2021; 40: 287-293

[8] Clemens V, Berthold O, Fegert J et al. Kinder psychisch erkrankter Eltern. Der Nervenarzt 2018; 89: 1262-1270

[9] DGPPN. S3 Leitlinie Psychosoziale Therapien bei schweren psychischen Erkrankungen. Berlin: DGPPN; 2018

[10] Mattejat F, Remschmidt $H$. The children of mentally ill parents. Deutsches Ärzteblatt International 2008; 105: 413
[11] Theule J, Wiener J, Tannock R et al. Parenting stress in families of children with ADHD: A meta-analysis. Journal of Emotional and Behavioral Disorders 2013; 21: 3-17

[12] Hayes SA, Watson SL. The impact of parenting stress: A meta-analysis of studies comparing the experience of parenting stress in parents of children with and without autism spectrum disorder. Journal of autism and developmental disorders 2013; 43: 629-642

[13] Gore FM, Bloem PJ, Patton GC et al. Global burden of disease in young people aged $10-24$ years: a systematic analysis. The Lancet 2011; 377: 2093-2102

[14] Frey M, Obermeier V, von Kries R et al. Age and sex specific incidence for depression from early childhood to adolescence: A 13-year longitudinal analysis of German health insurance data. Journal of psychiatric research 2020; 129: $17-23$

[15] Gillies D, Christou MA, Dixon AC et al. Prevalence and characteristics of self-harm in adolescents: meta-analyses of community-based studies 1990-2015. Journal of the American Academy of Child \& Adolescent Psychiatry 2018; 57: 733-741

[16] Paus T, Keshavan M, Giedd JN. Why do many psychiatric disorders emerge during adolescence? Nature reviews neuroscience 2008; 9 : 947

[17] Kessler RC, Avenevoli S, Costello EJ et al. Prevalence, persistence, and sociodemographic correlates of DSM-IV disorders in the National Comorbidity Survey Replication Adolescent Supplement. Archives of general psychiatry 2012; 69: 372-380

[18] Casey B, Duhoux S, Cohen MM. Adolescence: what do transmission, transition, and translation have to do with it? Neuron 2010; 67: 749-760

[19] Konrad K, Firk C, Uhlhaas PJ. Brain development during adolescence: neuroscientific insights into this developmental period. Deutsches Ärzteblatt International 2013; 110: 425

[20] Van Geel M, Vedder P, Tanilon J. Relationship between peer victimization, cyberbullying, and suicide in children and adolescents: a meta-analysis. JAMA pediatrics 2014; 168: 435-442

[21] Mullarkey MC, Marchetti I, Beevers CG. Using network analysis to identify central symptoms of adolescent depression. Journal of Clinical Child \& Adolescent Psychology 2019; 48: 656-668

[22] Bilsen J. Suicide and youth: risk factors. Frontiers in Psychiatry 2018; 9: 540

[23] DGKJP. S2k Leitlinie Nicht-suizidales selbstverletzendes Verhalten (NSSV) im Kindes- und Jugendalter. Berlin: DGKJP; 2016

[24] Bundesamt für Statistik. Todesursachen - Suizide 2019. Im Internet: https://www.destatis.de/DE/Themen/Gesellschaft-Umwelt/Gesundheit/Todesursachen/Tabellen/ suizide.html; Stand: 29.05.2021

[25] Kaess M, Parzer P, Haffner J et al. Explaining gender differences in non-fatal suicidal behaviour among adolescents: a population-based study. BMC Public Health 2011; 11: 597

[26] Kokkevi A, Rotsika V, Arapaki A et al. Adolescents' self-reported suicide attempts, self-harm thoughts and their correlates across 17 European countries. Journal of Child Psychology and Psychiatry 2012; 53: 381-389

[27] Brent DA, McMakin DL, Kennard BD et al. Protecting adolescents from self-harm: a critical review of intervention studies. Journal of the American Academy of Child \& Adolescent Psychiatry 2013; 52: 1260-1271

[28] Cluver L, Lachman JM, Sherr $L$ et al. Parenting in a time of COVID-19. Lancet 2020; 395 
[29] Guessoum SB, Lachal J, Radjack R et al. Adolescent psychiatric disorders during the COVID-19 pandemic and lockdown. Psychiatry research 2020; 113264

[30] Brown R, Witt A, Fegert JM et al. Psychosocial interventions for children and adolescents after man-made and natural disasters: a meta-analysis and systematic review. Psychological medicine 2017; 47: 1893-1905

[31] Ougrin D, Tranah T, Stahl D et al. Therapeutic interventions for suicide attempts and self-harm in adolescents: systematic review and meta-analysis. Journal of the American Academy of Child \&Adolescent Psychiatry 2015; 54: 97-107. e102

[32] Gulliver A, Griffiths KM, Christensen H. Perceived barriers and facilitators to mental health help-seeking in young people: a systematic review. BMC psychiatry 2010; 10: 1-9

[33] Fortune S, Sinclair J, Hawton K. Adolescents' views on preventing self-harm. Social psychiatry and psychiatric epidemiology 2008; 43: 96-104

[34] Xu Z, Huang F, Koesters $M$ et al. Effectiveness of interventions to promote help-seeking for mental health problems: systematic review and meta-analysis. Psychological Medicine 2018; 48: 2658-2667

[35] Zalsman G, Hawton K, Wasserman D et al. Suicide prevention strategies revisited: 10-year systematic review. The Lancet Psychiatry 2016; 3: 646-659

[36] Han J, Batterham PJ, Calear AL et al. Factors influencing professional help-seeking for suicidality: A systematic review. Crisis: The Journal of Crisis Intervention and Suicide Prevention 2018; 39: 175
[37] Marraccini ME, Brier ZM. School connectedness and suicidal thoughts and behaviors: A systematic meta-analysis. School Psychology Quarterly 2017; 32: 5

[38] Cox GR, Bailey E, Jorm AF et al. Development of suicide postvention guidelines for secondary schools: A Delphi study. BMC public health 2016; 16: 180

[39] Wharff EA, Ginnis KB, Ross AM et al. Family-based crisis intervention with suicidal adolescents: a randomized clinical trial. Pediatric emergency care 2019; 35: 170-175

[40] Frey LM, Hunt QA. Treatment for suicidal thoughts and behavior: A review of family-based interventions. Journal of marital and family therapy 2018; 44: 107-124

Bibliografie

Nervenheilkunde 2021; 40: 691-696

DOI 10.1055/a-1524-8628

ISSN $0722-1541$

(C) 2021. Thieme. All rights reserved.

Georg Thieme Verlag KG, Rüdigerstraße 14,

70469 Stuttgart, Germany 


\section{Punkte samneln auf CME.thieme.de}

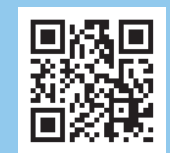

Diese Fortbildungseinheit ist bis zu 12 Monate online für die Teilnahme verfügbar.

Den genauen Einsendeschluss finden Sie beim Modul auf https://cme.thieme.de/CXHPZW6.

Sollten Sie Fragen zur Online-Teilnahme haben, finden Sie unter https://cme.thieme.de/hilfe

eine ausführliche Anleitung. Wir wünschen viel Erfolg beim Beantworten

der Fragen!

Unter https://eref.thieme.de/CXHPZW6 oder über den QR-Code kommen Sie

direkt zum Artikel.

VNR 2760512021160211193

\section{Frage 1}

Für welche psychische Störung im Kindes- und Jugendalter gibt es Hinweise, dass diese während der COVID-19-Pandemie zugenommen hat?
A Aufmerksamkeitsdefizit-/Hyperaktivitätsstörung (ADHS)
B Autismusspektrumsstörungen
C Essstörungen
D Somatoforme Störungen
E Dissoziative Störungen

\section{Frage 2}

Was ist unter den genannten Gründen der häufigste Grund für Inobhutnahmen bei Kindern $\leq 9$ Jahre?
A Sexueller Missbrauch
B Gefährdende Wohnverhältnisse
C Durch Eltern begangene Straftaten
D Drastische Beziehungsprobleme der Eltern
E Überforderung der Eltern

\section{Frage 3}

In welchem Alter sind die neurobiologischen Reifungsprozesse des Gehirns abgeschlossen?
A 12 Jahre
B 14 Jahre
C 18 Jahre
D 25 Jahre
E 30 Jahre

\section{Frage 4}

Was ist die ungefähre Lebenszeitprävalenz von Suizidversuchen bei jugendlichen Mädchen?
A $0,1 \%$
B $1 \%$
C $2 \%$
D $5 \%$
E $10 \%$

\section{Frage 5}

Was zählt zu den 3 wichtigsten Risikofaktoren für einen Suizidversuch im Jugendalter?
A Schulabsentisums
B Suizidversuche in der Vorgeschichte
C Broken-Home-Situation
D Cannabisabhängigkeit
E Leistungsknick in der Schule

\section{Frage 6}

Was ist für Jugendliche mit psychischen Erkrankungen eine häufige Hürde für die Inanspruchnahme von professioneller Hilfe?
A Angst vor Stigmatisierung
B Fehlinformation durch Gleichaltrige
C Abhängigkeitsverhältnis zu den Eltern
D Fehlen passender Hilfsangebote
E Hohe Anzahl an Komorbiditäten

\section{Frage 7}

Welche Maßnahmen an Schulen zur Suizidprävention sind in ihrer Wirksamkeit gut durch Studien belegt?
A Schulung und Einsatz von Gatekeepern
B Screening-Maßnahmen für psychische Erkrankungen und Suizidalität
C Aufklärung zu psychischen Erkrankungen und Suizidalität
D Internetforen für Schüler zum Thema psychische Erkrankungen
E Serious Games zum Thema psychische Erkrankungen

\section{Frage 8}

Was ist ein häufiger Belastungsfaktor für Kinder psychisch kranker Eltern?
A Erlebte Verpflichtung zum Schweigen
B Unterernährung
C Konflikte in der Peergroup
D Geschwisterrivalität
E Fehlende Routinen im Alltag 


\section{Punkte sammeln auf CME.thieme.de}

Fortsetzung $\ldots$

\section{Frage 9}

Welcher Kombination an neurobiologisch zeitlich versetzt reifenden Strukturen wird eine Förderung der Eigenschaften „Flexibilität“ und „Offenheit für Neues“ in der adoleszenten Entwicklung nachgesagt?
A Anteriorer zingulärer Kortex und Hypothalamus
B Limbisches System und präfrontaler Kortex
C Hippocampus und Amygdala
D Fornix und Nucleus accumbens
E Präfrontaler Kortex und Inselrinde

\section{Frage 10}

Welche psychiatrischen Erkrankungen im Kindesalter sind ein häufiger Anlass für krisenhafte Zuspitzungen aufgrund einer Überforderung der Eltern?
A Enkopresis und Enuresis
B Schizophrenie
C ADHS
D Dissoziative Störungen
E Parasomnien 